Musées, Patrimoine et Culture scientifiques et techniques

$183 \mid 2019$

mai-juin 2019

\title{
Exposer les sujets sensibles : comment et pour qui ?
}

\section{Marie-Sylvie Poli}

\section{OpenEdition \\ Journals}

Édition électronique

URL : http://journals.openedition.org/ocim/2435

DOI : $10.4000 /$ ocim. 2435

ISSN : 2108-646X

Éditeur

OCIM

Édition imprimée

Date de publication : 1 mai 2019

Pagination : 10-17

ISSN : 0994-1908

Référence électronique

Marie-Sylvie Poli, « Exposer les sujets sensibles : comment et pour qui ? », La Lettre de I'OCIM [En ligne], 183 | 2019, mis en ligne le 01 juin 2020, consulté le 22 mars 2021. URL : http://

journals.openedition.org/ocim/2435; DOI : https://doi.org/10.4000/ocim.2435

Ce document a été généré automatiquement le 22 mars 2021.

Tous droits réservés 


\title{
Exposer les sujets sensibles : comment et pour qui ?
}

\author{
Marie-Sylvie Poli
}

L'exposition Terra Data nos vies à l'ère du numérique (2017-2018) ou comment protéger nos données personnelles face aux dispositifs invasifs numériques.

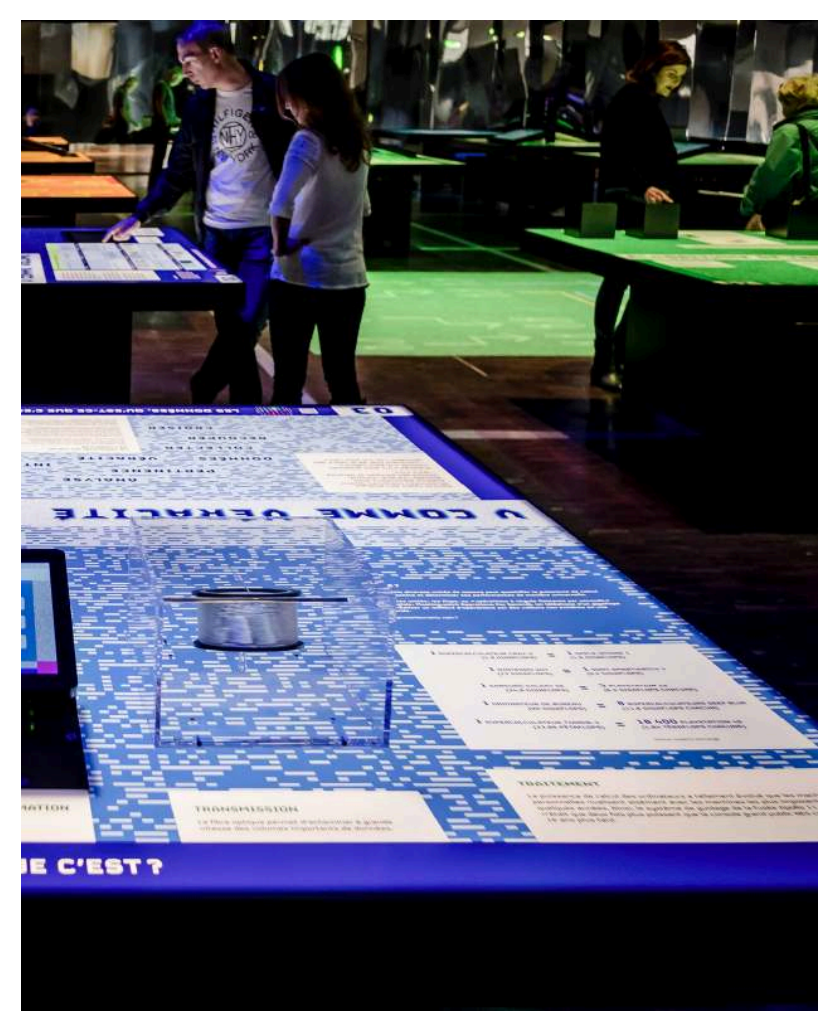

(C) Cité des Sciences et de l'Industrie

1 Partons du postulat qu'un "sujet sensible " est une thématique à la fois intime et universelle, mise en débat par la société en dehors du musée. Lorsqu'on réfléchit en muséologue à ce que signifie "exposer les sujets sensibles", seule la "hot 
museology " nord américaine explicite les enjeux culturels et les règles de vigilance à convoquer et à respecter pour exposer des sujets sensibles.

\section{Dans sensible il y a sens}

2 Certaines visites d'expositions restent comme une expérience intense qui nous a touchés dans nos valeurs, dans notre vision du monde, dans nos croyances, dans nos certitudes. Nous y avons été sensible car elle faisait sens pour nous.

3 C'était parfois une exposition au budget modique portée par une sobre scénographie à laquelle les médias ne s'étaient pas intéressé ou au contraire, un évènement d'envergure doté de dispositifs technologiques dispendieux créés à l'occasion pour flatter des collections agencées par une scénographie raffinée. Cette exposition, temporaire ou de longue durée, se passait parfois dans un musée d'art ou d'histoire et de société, tantôt dans un musée de sciences ou un tout autre lieu comme une friche, un centre de culture technique. Tantôt ce sont les textes, les témoignages, les visages, tantôt les modes de médiation qui nous ont émus.

4 Comment expliquer que certaines expositions nous émeuvent plus que d'autres, nous frappent durablement, nous amènent à plus de sens critique ?

5 Première hypothèse, ces expositions traitent d'une question particulière parce que jugée «sujet sensible » par tout public. On dira qu'il s'agit d'un «sujet sensible » du point de vue de la réception sociétale.

6 Seconde hypothèse, c'est parce que le sujet qu'elles exposent nous touche intimement dans notre histoire personnelle. On dira qu'il s'agit d'un « sujet sensible » du point de vue de la réception individuelle.

7 C'est la tension entre ces deux hypothèses qui sera discutée ici. Nous décrivons d'abord les modalités spécifiques d'écriture expographique d'expositions qui traitent de sujets sensibles, leurs mécaniques discursives et les postures énonciatives de leurs auteurs.

8 Après quoi nous présentons le cadre méthodologique de recherches en muséologie de la réception compréhensive pour traiter ce questionnement.

9 La conclusion est l'occasion d'appréhender les enjeux politiques de ce type d'expositions exigeantes et difficiles, autant pour les publics que pour les institutions et tous les professionnels qui en portent la responsabilité.

\section{Les modalités de l'écriture expographique du sujet sensible}

10 Le calendrier des commémorations, un projet de rénovation de bâtiments ou de salles, la commande d'un mécène ou d'un partenaire institutionnel, l'intérêt d'un conservateur pour des thèmes non encore traités au musée et bien d'autres raisons encore peuvent amener les équipes d'une institution muséale à devoir traiter de sujets qu'on dit généralement sensibles. Sensibles parce qu'ils abordent des questions qui touchent aux valeurs sociales ou communautaires, aux sentiments de chacun, aux tabous qui fondent notre socle anthropologique, à des problèmes politiques ou culturels qui agitent la société contemporaine. Une chose est certaine, ces expositions posent des problèmes aux professionnels qui sont en charge de les réaliser; pas 
seulement des problèmes de contenus, de mise en forme ou de muséographie auxquels ils sont aguerris. Ce type d'exposition les alerte sur la question de la vigilance éthique à maintenir vis-à-vis des publics qui, dans la diversité de leur âge, de leur culture, de leur sensibilité, peuvent se sentir offensés par ce que l'exposition montre, dit, ne dit pas, comment elle montre, comment elle dit, pourquoi elle ne dit pas.

Début du parcours de l'exposition Spoliés ! L'« aryanisation » économique en France 1940-1944

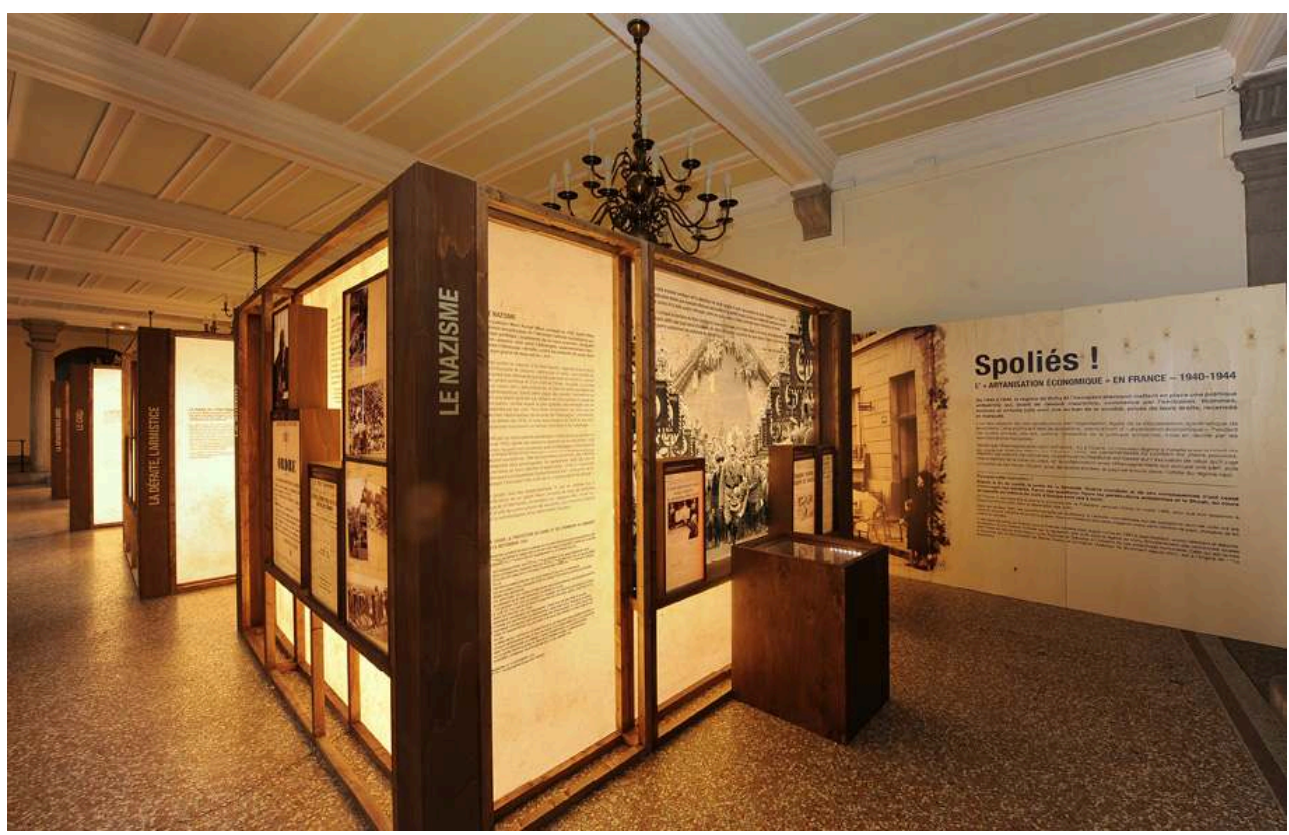

(C) Musée de la Résistance et de la Déportation de l'Isère

Peut-on dresser une typologie des modalités de conception de ces expositions qui exposent des sujets sensibles tels que définis ci-avant? La tache est ardue car il est illusoire de réduire ces expositions à des types de sujets et à des mécaniques d'écriture standardisées. Toutefois il est possible de proposer des hypothèses sur ce qui caractérise le traitement satisfaisant d'un sujet sensible par l'exposition.

\section{S'émanciper du discours médiatique dominant}

12 Il est nécessaire que les concepteurs de l'exposition se positionnent autrement que les médias de masse sur le sujet, même s'ils intègrent certaines de leurs représentations, certaines de leurs informations.

13 Les muséographes ne peuvent passer outre les discours des médias. Mais pour répondre à son devoir d'exigence et de neutralité, le musée doit s'en détacher. Comme sa mission l'y engage, il doit traiter tout sujet en s'appuyant sur des sources scientifiques multiples, validées par des experts. Il est conforté dans cette exigence par les publics dont on connaît désormais la profonde méfiance envers les médias de masse et envers les réseaux sociaux. Même si tout un chacun les pratique, plus ou moins assidument.

14 C'est pourquoi s'il veut rester crédible, le musée doit proposer une vision critique sur tout sujet à débat, une vision en rupture avec celle des médias. Ce qui impose aux équipes de conception de réaliser une intense veille médiatique et sur les réseaux sociaux. 

elle pas à un collectif anonyme, mais à des professionnels (curateurs, scientifiques, artistes, commissaires, conservateurs, médiateurs) nommés dans les dispositifs comme l'ours, les panneaux, les cartels, les dispositifs audio et vidéo, les supports numériques comme le site du musée et autres. Plus fondamentalement cette pratique collaborative permet à tous les acteurs du projet de développer à partir de chaque exposition sur un sujet sensible, une expérience réflexive sur le rôle politique du champ muséal, de sa propre institution.

21 Car exposer un sujet sensible impose de considérer les objets des collections comme les arguments d'une démonstration pour servir le propos expographique, pour soutenir la thèse défendue par l'exposition. La rhétorique pragmatique de ce type d'exposition (qui doit toucher le visiteur) se traduit au niveau du contenu par un propos à la fois polyphonique et assumé, et au niveau de la forme, par une mobilisation inédite des patrimoines matériels et immatériels exposés.

22 Car l'exposition sur un sujet sensible agit sur le visiteur par le verbe, par l'image, par l'ambiance, mais aussi par l'agencement et la mise en scène des patrimoines. 
Ce dimension humaine et politique de la forme exposition va se retrouver déclinée dans tous les autres dispositifs d'escorte comme le catalogue, les produits dérivés, les outils de communication papier et numérique, le dossier pédagogique, les annonces sur les réseaux sociaux, et bien sûr les discours des professionnels qui en assurent la médiation auprès de tous les publics.

Panneau consacré à l'aryanisation dans l'exposition Spoliés !

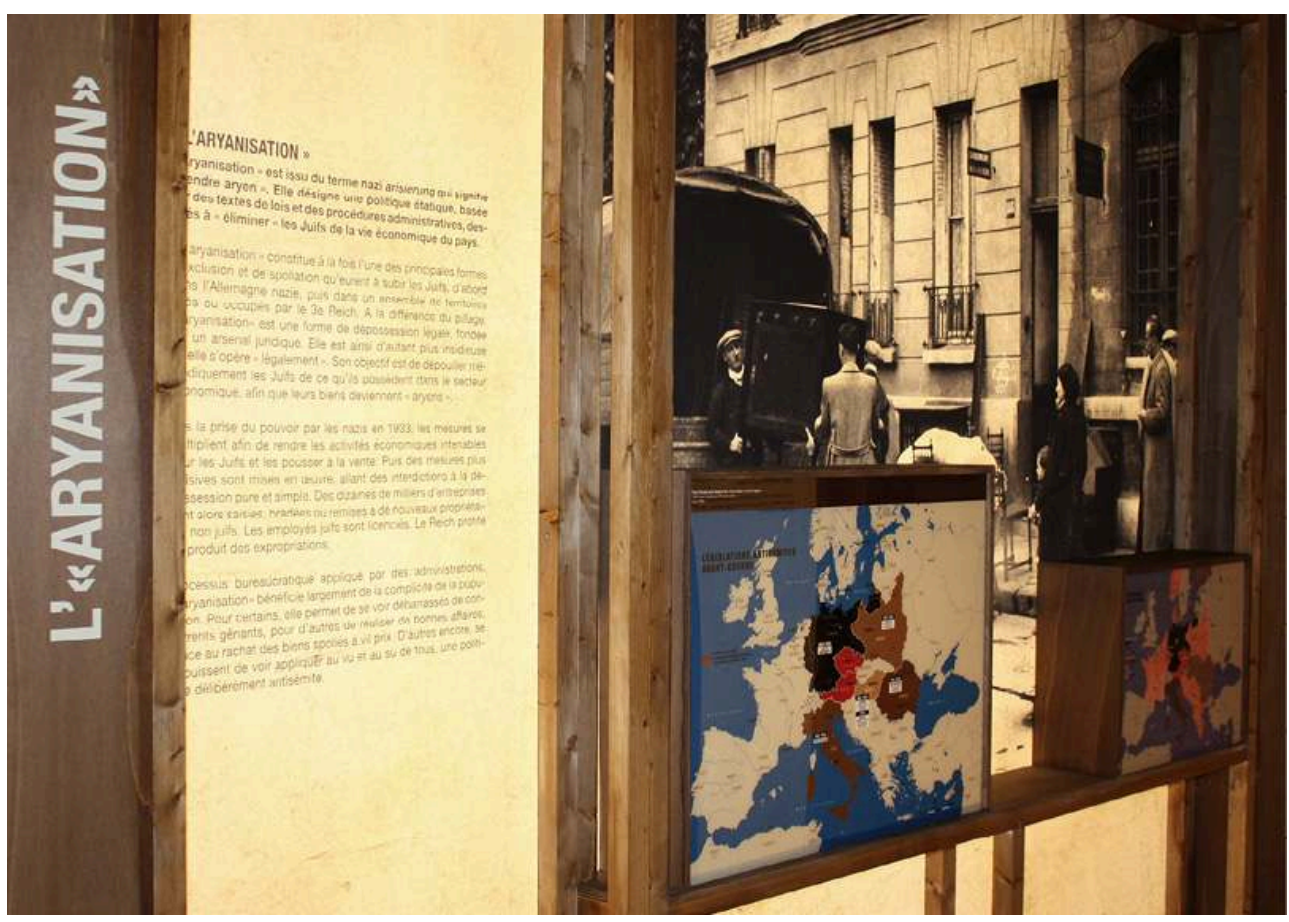

(C) Musée de la Résistance et de la Déportation de l'Isère

\section{Rester vigilant à ne pas exclure certains publics}

Comment ne pas choquer? Ne pas froisser les identités tout en parlant vrai puisque ce type d'exposition peut ne pas plaire à tous les visiteurs ? Certes les lignes de vigilance à identifier sont nombreuses et différentes pour chaque sujet. Les précautions à prendre sont mouvantes en fonction des profondes transformations culturelles de notre société, distinctes selon les territoires, leurs mémoires et leurs projets politiques.

Certaines expositions sur des sujets sensibles vont défrayer la chronique, être auréolées d'une odeur de souffre et de scandale alors qu'elles cherchent seulement à étonner. D'autres passeront inaperçues alors qu'elles font œuvre d'un réel courage.

Dans un pays où la censure épargne le champ muséal, ce sont les critiques et les visiteurs qui dictent les limites de la sensibilité à ne pas dépasser. On peut compter sur le public pour fixer certaines lignes de vigilance; reste aux professionnels des musées d'y être attentifs puis de décider où placer le curseur sans pour autant se désavouer.

Les médiateurs qui se trouvent au contact direct avec les visiteurs jouent donc un rôle essentiel de caisse de résonance des effets produits par l'exposition car ils entendent les visiteurs exprimer leur malaise, leur gêne, parfois leur colère. Une autre manière de prendre en compte cette délicate question des lignes de vigilance à mettre en place 
pour exposer un sujet sensible, consiste à pratiquer ou à faire pratiquer des études de réception rigoureuses du point de vue méthodologique.

C'est l'objet majeur de la muséologie de la réception compréhensive. Voyons avec quelles méthodes, pour quels résultats.

\section{Entrée de l'exposition Terra Data nos vies à l'ère du numérique}

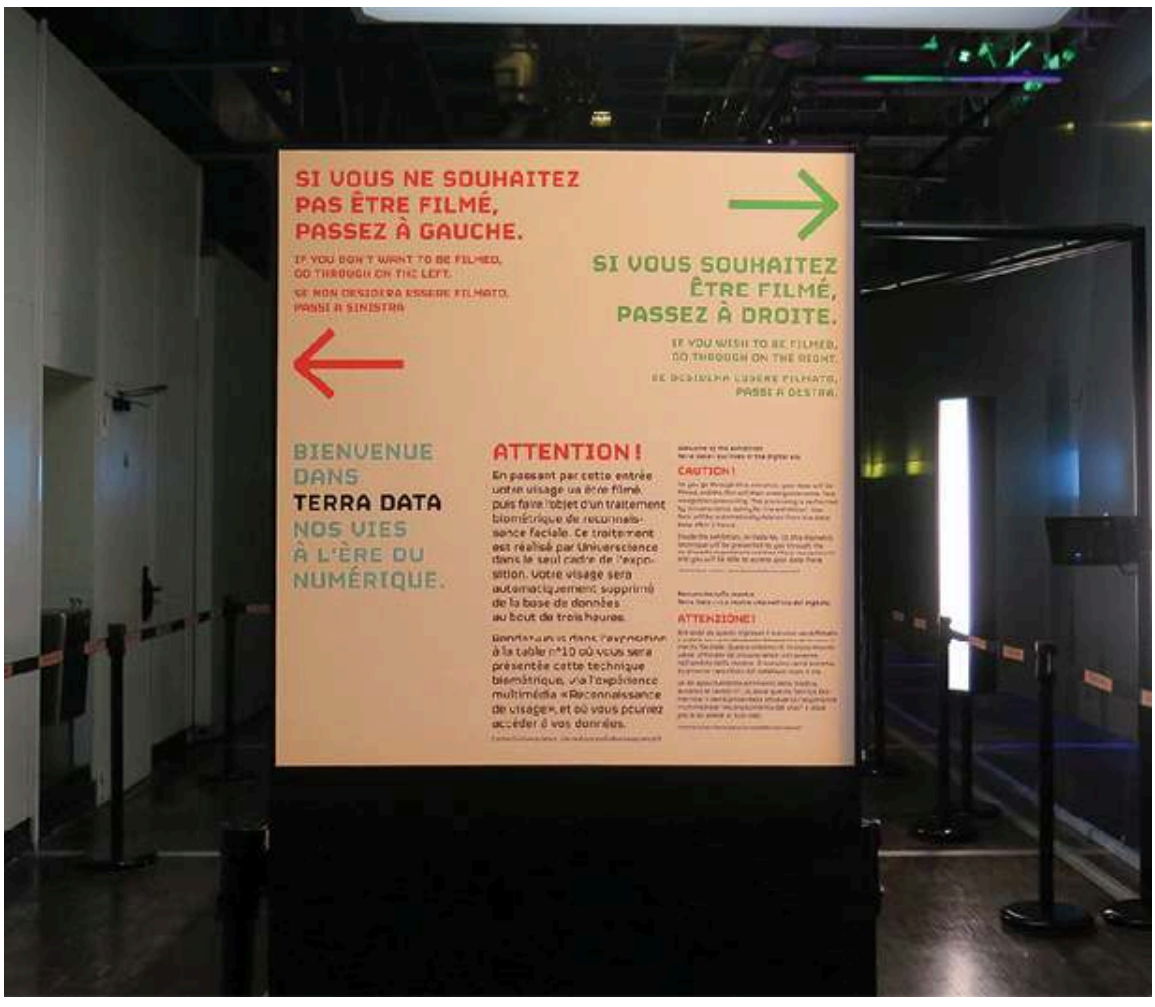

(c) Cité des Sciences et de l'Industrie

\section{Les apports de la muséologie sociale et compréhensive}

Cette muséologie s'intéresse avant tout au sens que les gens donnent à leurs interactions avec l'exposition en s'appuyant sur des enquêtes de terrain et sur la prise en compte de leurs récits d'expérience de visite. Lors d'entretiens non directifs le chercheur ne questionne pas seulement les appréciations de ce que les gens viennent de voir et d'entendre, il les invite à se positionner sur le sujet exposé. Puis grâce à des analyses de discours de ces récits d'expérience, le chercheur étudie les motivations préalables à la visite de l'exposition ainsi que l'intensité de ses retentissements après la visite.

L'analyse sémantique des propos des interviewés ${ }^{1}$ permet d'établir les listes des univers de référence des paroles de l'interviewé, c'est-à-dire de créer le relevé de tous les champs sémantiques mobilisés dans le récit d'expérience de visite. Ce qui est plus intéressant qu'une simple liste de mots lorsqu'il s'agit de comprendre la complexité psychologique des paroles du visiteur. Car l'ensemble des univers de sens ainsi répertoriés indique précisément les problématiques évoquées par le visiteur à propos 
de tous les composants de l'exposition. Une fois que tous les univers de sens sont répertoriés ils sont ensuite analysés selon leur valeur sémantique, leur pondération statistique, leurs contextes d'apparition (moments dans l'entretien, récurrence) et leur dimension émotive. On parvient ainsi à focaliser les analyses sur tel ou tel topic, dont le sujet de l'exposition.

31 Après que tous les univers de sens qui désignent le sujet de l'exposition dans l'entretien ont été répertoriés et étudiés, on est en mesure de saisir l'importance que chaque visiteur accorde au sujet de l'exposition mais aussi comment il exprime le rapport plus ou moins intime, plus ou moins distancié qu'il établit avec ce sujet.

Loin d'être exprimées de manière logique et univoque, ces évocations libres du sujet de l'exposition donnent lieu à des avis contrastés, à des incohérences, à des paradoxes qui dénotent la distance ou au contraire l'émoi, parfois le malaise dans lesquels le sujet de l'exposition place le visiteur.

Ces indicateurs linguistiques de troubles vécus lors de l'expérience de visite permettent alors au chercheur d'évaluer si pour le visiteur interrogé, le sujet de l'exposition est réellement un sujet sensible.

L'enjeu de ces recherches de terrain menées sur des expositions dissemblables en tout (thèmes, muséographie, médiation, calendrier, tutelles, conception, budget) est majeur. Car avec cette méthodologie d'enquête qualitative il s'agit de se départir des représentations médiatiques ou idéologiques communes de ce qu'est un sujet sensible, pour proposer une acception strictement muséologique de la notion de sujet sensible dans le champ muséal aujourd'hui, du point de vue des publics.

Afin de concrétiser cette démarche compréhensive, nous allons nous référer à trois recherches de terrain réalisées sur trois expositions $a$ priori très dissemblables, mais que les visiteurs ont vécues et reconnues comme exposant un sujet sensible.

\section{Sujet sensible et exposition de beaux-arts}

De mars à juin 2005, le musée de Grenoble propose d'étudier l'évolution de la peinture métaphysique avec l'exposition L'art italien et la metafisica - Le temps de la mélancolie 1912-1935. La majorité des 600 visiteurs enquêtés disent avoir saisi les interférences entre la montée du fascisme et l'évolution de la peinture vers une représentation formelle austère, sombre, silencieuse et figée de la ville, de la famille, de la nature.

Mais pour certaines personnes cette exposition évoque un tout autre sujet sensible que cette période de l'histoire de l'art italien. Elle leur permet de se remémorer les récits poignants des conditions de détresse dans lesquelles leurs aïeuls quittèrent dans les années 1910-1930, une Italie en proie à la montée des extrémismes politiques et religieux.

Les textes des aides à la visite et les écrits des philosophes bouleversent certains visiteurs en donnant du sens aux non-dits qui entourent encore le silence gêné de leurs familles à propos des véritables raisons de leur venue en France : la famine, la pauvreté, le chômage, la violence familiale et sociale.

Pour d'autres La metafisica met à mal les clichés de l'Italie du bel canto, de la lumière, de la joyeuse beauté des villes.

Quelques personnes expliquent que l'exposition les touche car elle leur permet de poser un regard critique sur l'engagement en art, sur le courage politique de certains artistes. 

arrivant en Italie en 2005 et les épreuves de leurs grands-parents, immigrant du sud de l'Italie pour venir vivre à Grenoble au début du XX' siècle.

Le plateau de l'exposition Terra Data nos vies à l'ère du numérique

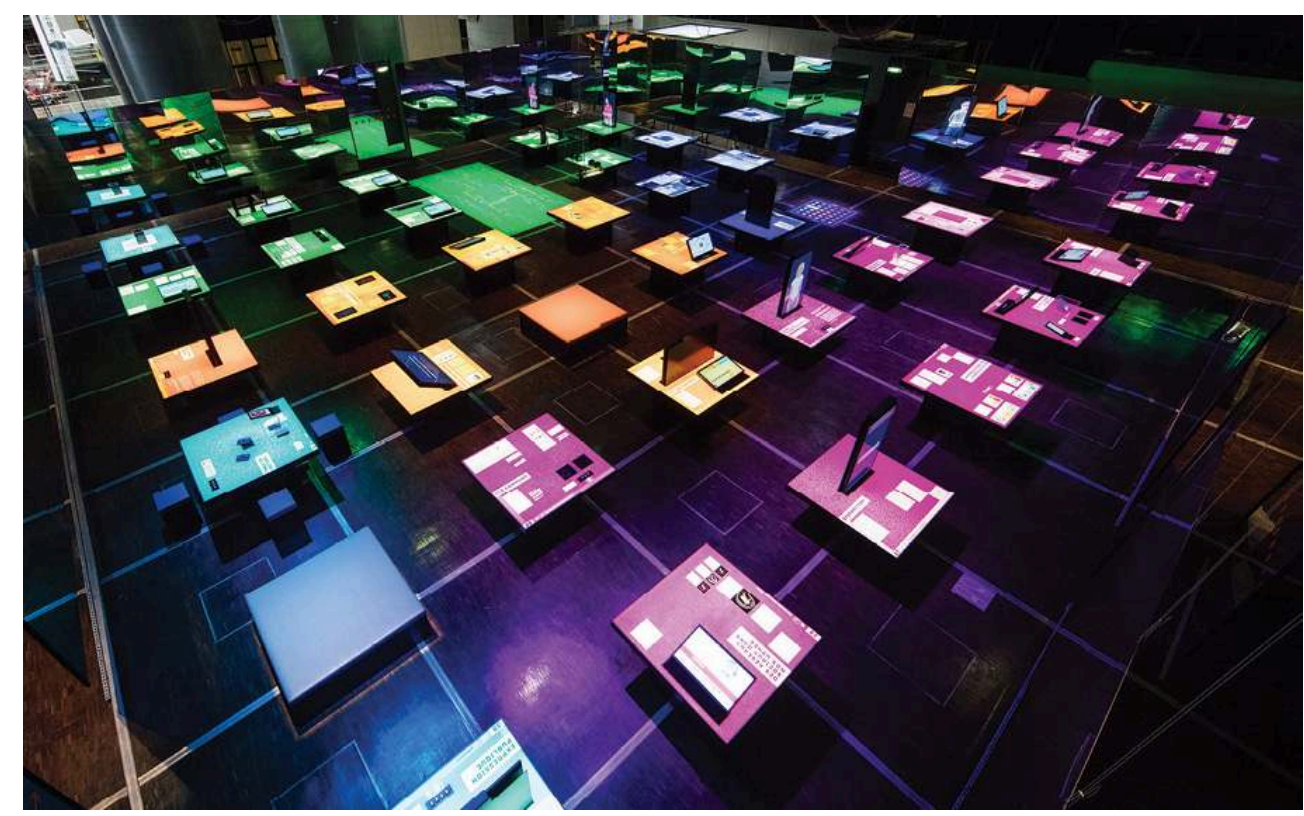

(C) Cité des Sciences et de l'Industrie

\section{Sujet sensible et exposition d'histoire}

Nous avons réalisé une étude compréhensive sur la réception de Spoliés! L'« aryanisation " économique en France 1940-1944 conçue par le musée de la Résistance et de la Déportation de l'Isère à Grenoble et présentée hors les murs au Palais du Parlement du Dauphiné de juin 2010 à février 2011². Spoliés! traite dans une perspective historique et régionale de la spoliation des Juifs en Isère pendant la Seconde Guerre mondiale.

Environ 600 questionnaires sont administrés auprès des visiteurs. Ils dénotent l'intérêt porté aux documents d'archives, aux contenus historiques qui divulguent les implications de la police et des civils dans le double mécanisme de dénonciation et de spoliation de familles juives en Isère vivant dans une grande précarité économique. Une quarantaine de personnes acceptent de livrer leurs ressentis au cours d'entretiens non directifs. Leurs analyses méritent toute notre attention.

44 Ces analyses nous apprennent que ce n'est pas la spoliation des Juifs qui représente un sujet sensible pour certains visiteurs, mais le fait que cette exposition mette à mal l'image de Grenoble ville résistante, honorée comme telle par le Général de Gaulle (Croix de la Libération remise à la ville le 5 novembre 1944).

45 Le discours de l'exposition heurte leur vision de Grenoble et par conséquent remet en cause leur fierté d'être Grenoblois. Pour ces personnes, le sujet sensible de Spoliés! c'est la manière dont selon eux, elle déshonore la mémoire des habitants de leur ville pendant ces années de guerre. Ils estiment que l'exposition ne transmet pas la vérité historique. 
D'autres au contraire, se disent bouleversés par les documents d'archives qui prouvent que des entreprises et des citoyens ordinaires s'engagèrent volontairement dans les opérations de l'«aryanisation" en Isère. Pour ces personnes, le sujet sensible de Spoliés ! c'est la question de l'éthique et du courage personnel en période de guerre ; aussi se demandent-ils avec sincérité s'ils auraient été courageux ou lâches, collaborateurs ou antisémites actifs.

Pour certains enfin Spoliés! invoque le conflit entre Palestine et Israël aujourd'hui. Ils regrettent que les musées d'histoire en général et le musée de la Résistance et de la Déportation de l'Isère en particulier ne traitent pas ce sujet alors qu'il est selon eux abordé par les médias en favorisant les positions sionistes. Pour ces personnes, le sujet sensible de Spoliés!est plus largement une remise en question des missions traditionnelles du musée d'histoire et leur revendication que ce type de musée s'engage à donner sa lecture des conflits nationaux et internationaux en cours.

\section{Sujet sensible et exposition de science et technique}

8 Passons à l'exposition de science et technique avec Terra data nos vies à l'ère du numérique présentée d'avril 2017 à janvier 2018 à la Cité des Sciences et de l'Industrie à Paris $^{3}$. Les récits d'expérience de visite montrent que la dimension technique et immersive de la muséographie a convaincu. Le thème et les enjeux de l'exposition sont compris : la question de la défense de nos libertés individuelles et de notre intimité à une période où nous confions naïvement nos données personnelles par internet à des plateformes d'intermédiation marchandes et à des réseaux sociaux numériques dont nous ne maitrisons aucune modalité de fonctionnement informatique et juridique.

Toutefois pour certains visiteurs, le sujet sensible est ailleurs, Terra data traite de notre dépendance psychologique à des dispositifs invasifs comme les téléphones mobiles intelligents, les tablettes numériques, les ordinateurs.

D'autres vivent mal le fait que l'exposition leur suggère que pour se "libérer » il leur faut consacrer beaucoup de temps, d'intelligence et d'énergie à se former au droit et à l'informatique; un défi qu'ils ne se sentent pas capables de relever.

Pour quelques-uns l'exposition renforce les clichés qui présentent les personnes de plus de cinquante ans dépassées, incapables de maîtriser les technologies du numérique et le problème de la gestion des data.

Enfin elle est appréhendée comme un sujet politique sensible par les visiteurs pour lesquels l'État, l'école et les musées devraient de toute urgence se mobiliser pour que tout un chacun puisse gouverner la manière dont il veut que les acteurs du numérique aient ou non accès à ses data. 
Les visiteurs confrontés aux « Panama Papers » dans l'exposition Terra Data nos vies à l'ère du numérique

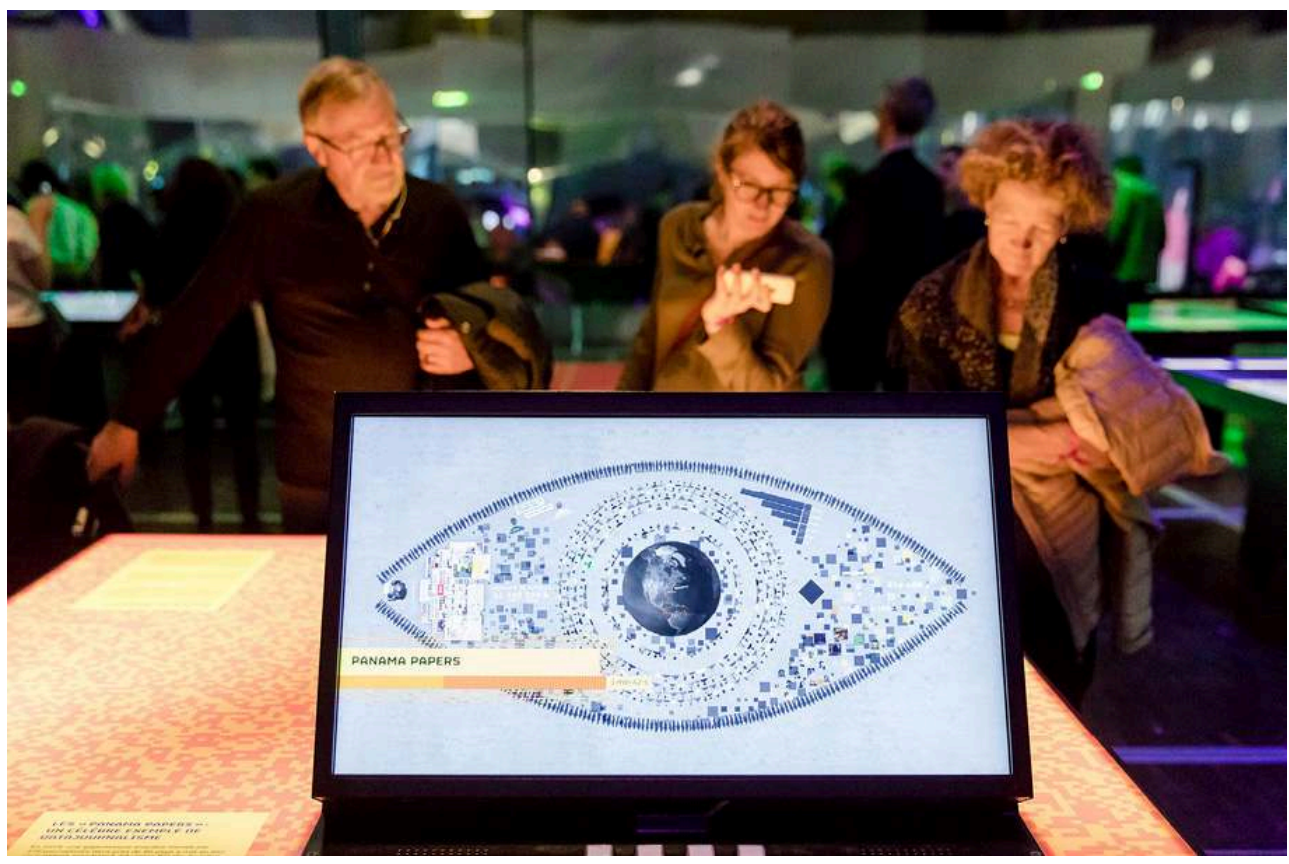

(c) Cité des Sciences et de l'Industrie

\section{Inviter le visiteur à l'analyse critique de notre société}

Nous nous en tiendrons à ces trois exemples mais nous pourrions multiplier les recherches en réception compréhensive qui prouvent que c'est le visiteur et lui seul, qui décide si le sujet d'une exposition est, ou n'est pas pour lui, un sujet sensible.

L'intrusion croissante dans les musées de l'internet et des réseaux sociaux ne doit pas seulement être perçue comme une contrainte technologique à se conformer aux modes de communication du temps présent. Car l'enjeu muséal de cette métamorphose communicationnelle va bien au-delà d'une seule instrumentation numérique des fonctions de transmission des savoirs. Cette transformation vers plus d'intermédiation publics/musée annonce la volonté de certains musées de s'emparer de sujets qui sont précisément discutés sur les réseaux sociaux et dans les médias, entre scientifiques et au plus haut niveau de nos institutions politiques (réchauffement climatique, genre et pouvoir, maladie, mort, immigration, chômage, et tant d'autres sujets à controverse).

Aussi faut-il considérer la multiplication d'expositions qui prennent le risque d'exposer des sujets sensibles comme une nouvelle forme d'engagement du musée vis-vis de la société. Un engagement bel et bien politique qui, s'il se confirme, permettra à l'avenir que les publics viennent sciemment au musée pour participer, par leur analyse critique, aux débats sur les grandes questions du temps. 


\section{BIBLIOGRAPHIE}

Cabanas, E. et Illouz, E. Happycratie. Comment l'industrie du bonheur a pris le contrôle de nos vies. Clermont-Ferrand : Premier Parallèle, 2018, 269 p.

Daignault, L. et Schiele, B. (dir.) Les musées et leurs publics : savoirs et enjeux. Québec : Presses de l'Université du Québec, 2014, 362 p.

Eidelman, J. et Roustan, M. (dir) La place des publics. De l'usage des études et recherches par les musées. Paris : La Documentation française, 2007, 334 p.

Poli, M.-S. et Ancel, P. Exposer l'histoire contemporaine. Évaluation muséologique d'une exposition : Spoliés! L' «aryanisation » économique en France 1940-1944. Paris : La Documentation française. 2014, $95 \mathrm{p}$.

\section{NOTES}

1. Nous utilisons le logiciel d'analyse de discours Tropes.

2. www.resistance-en-isere.fr/2293-spolies-l-aryanisation-economique-en-france-1940-1944.htm

3. www.cite-sciences.fr/fr/ressources/expositions-passees/terra-data/

\section{RÉSUMÉS}

L'auteure propose une double approche des expositions qui traitent des sujets sensibles. Elle questionne d'une part leur écriture, qui nécessite une vigilance éthique, une vision critique du sujet et la nécessité de propos clairs assumés et d'autre part leur réception dont le degré diffère en fonction des visiteurs ou groupes de visiteurs considérés.

\section{INDEX}

Mots-clés : Exposition, visiteurs, éthique

\section{AUTEUR}

\section{MARIE-SYLVIE POLI}

Professeur émérite à Avignon Université, Centre Norbert Elias, équipe Culture et Communication marie-sylvie.poli@univ-avignon.fr 